\title{
Disseminated Tuberculosis Associated With Adalimumab Therapy
}

\author{
Marinha Silva ${ }^{\mathrm{a}, \mathrm{b}}$, Joana Braga ${ }^{\mathrm{a}}$, Cristiana Fernandes ${ }^{\mathrm{a}}$, Jose Miguel Ferreira ${ }^{\mathrm{a}}$, \\ Cristina Marques ${ }^{\mathrm{a}}$, Carina Costa Silva ${ }^{\mathrm{a}}$, Carla Lemos Costa ${ }^{\mathrm{a}}$
}

\begin{abstract}
Therapeutic blockade of tumour necrosis factor alpha (anti-TNF- $\alpha$ ) is the mainstay treatment of several rheumatologic diseases. They are unfrequently associated with opportunistic infections and latent tuberculosis (TB) screening is paramount before immunosuppression. A 62-year-old man with psoriatic arthritis was under treatment with adalimumab for over 5 years. The screening for latent tuberculosis infection (LTBI) prior to the start of immunosuppression was negative, and a subsequent interferon gamma release assay 4 years later was also negative. He presented in the emergency department complaining of asthenia, anorexia, fever, night sweats and weight loss for over 5 months. He also complained of memory loss, despite his normal cognitive and neurological examination. After an exhaustive workup, he was diagnosed with a disseminated tuberculosis (cerebral, pulmonary and peritoneal) and treated accordingly. TB diagnosis remains challenging in certain situations. Latent TB screening tests may lack sensitivity and immunosuppressive therapy increases the risk of disseminated infection. We discuss the workup and management of a central nervous system disseminated TB related to adalimumab therapy.
\end{abstract}

Keywords: Adalimumab; Tuberculosis; Psoriatic arthritis; Tuberculomas

\section{Introduction}

Therapeutic blockade of tumor necrosis factor alpha (antiTNF- $\alpha$ ) is the treatment of multiple autoimmune diseases, with marked improvement in the quality of life of patients. They have a good safety profile. However, they increase the risk of infections, especially of the respiratory and urinary tract. The risk of tuberculosis (TB) in these patients is higher than in the

Manuscript submitted May 29, 2021, accepted June 12, 2021

Published online August 25, 2021

anternal Medicine Department, Hospital Santa Maria Maior, Barcelos, Portugal

${ }^{b}$ Corresponding Author: Marinha Silva, Rua Padre Francisco Rodrigues, no. 1880, 4800-606, Santa Eufemia de Prazins, Guimaraes, Portugal.

Email: marinhasilva@gmail.com

doi: https://doi.org/10.14740/jmc3723 normal population, with Mycobacterium tuberculosis (MB) being the most common agent. If TB develops during anti-TNF- $\alpha$ treatment, it may present as disseminated or extrapulmonary. In Portugal, TB incidence is 16.6 cases per 100,000 people.

\section{Case Report}

\section{Investigations}

A 62-year-old overweight man with past history of arterial hypertension, type 2 diabetes mellitus, dyslipidemia and psoriatic arthritis was under treatment with adalimumab for 5 years. The pre-treatment interferon gamma release assay (IGRA) in 2012 was negative, as well as a 2018 IGRA test. The tuberculin skin test (TST) was not performed at the time and the patient did receive the bacillus Calmette-Guerin vaccine in his infancy. His brother was diagnosed with bone TB in 2012 when the patient was already under adalimumab.

He presented to the emergency department in November 2019 with constitutional symptoms of asthenia, anorexia, low grade fever, night sweats and weight loss progressing over 5 months.

On initial assessment, he was hemodynamically stable, but subfebrile with pale mucous membranes. Cardiac and pulmonary auscultations were normal but there was tenderness on abdominal palpation on the flanks suggesting ascites. The neurological examination was normal.

\section{Diagnosis}

The hemogram showed a normal blood count, elevated sedimentation velocity $(90 \mathrm{~mm} / \mathrm{h})$ and C-reactive protein $(9.56$ $\mathrm{mg} / \mathrm{dL}$ ), with normal renal, thyroid and hepatic function tests, negative viral serology and normal levels of immunoglobulins. The X-ray and chest computed tomography (CT) scan revealed a bilateral micronodular pattern (white arrow) and evidenced multiple adenopathies (red arrow, Fig. 1). He was admitted to the ward and adalimumab therapy suspended. During hospitalization, abdominal-pelvic CT showed high-volume ascites with heterogeneity and densification of the peritoneum (Fig. 2). Adenosine deaminase (ADA) in the ascites was 2.5 times higher than normal on diagnostic paracentesis.

He underwent a videobronchofibroscopy with bronchoal- 


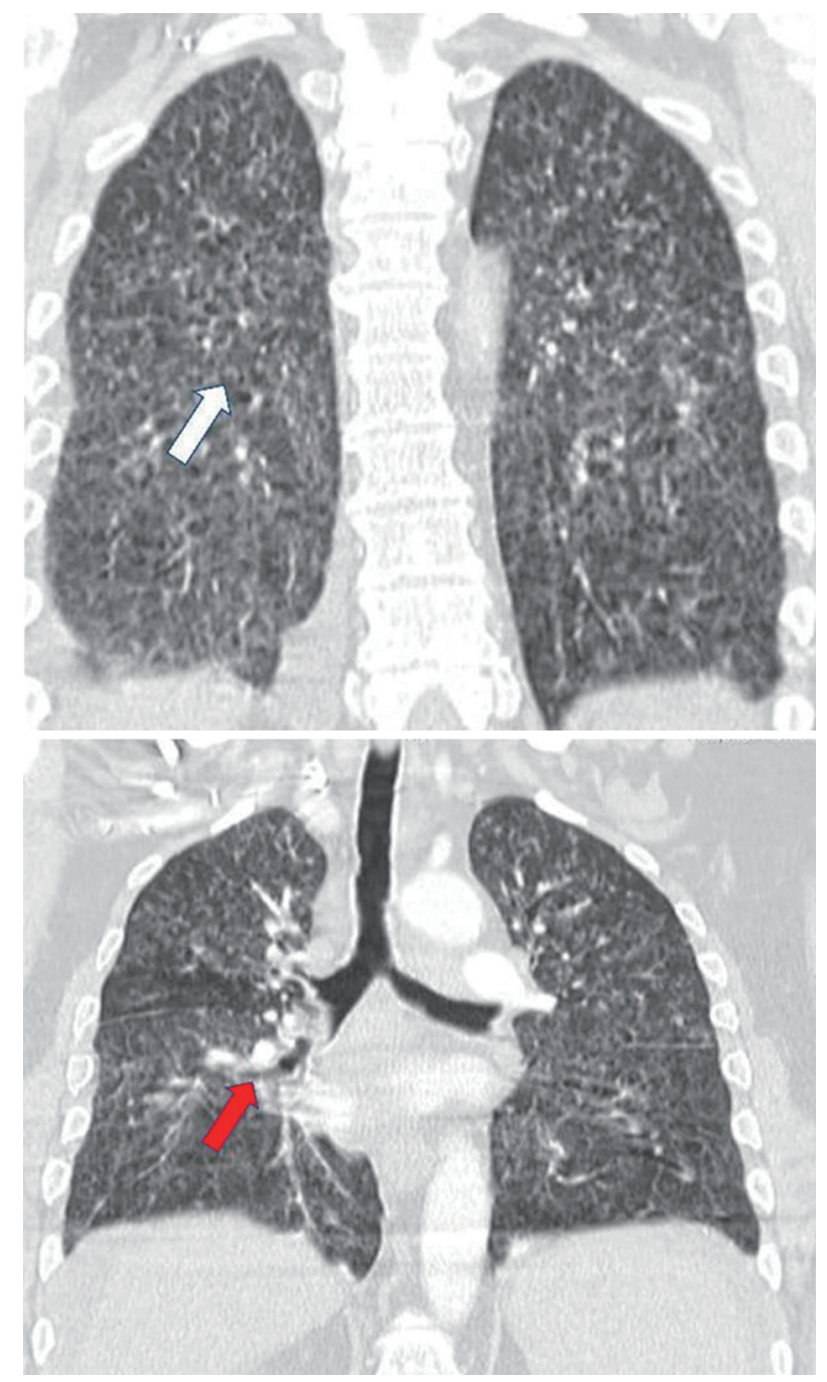

Figure 1. Thoracic CT showing bilateral micronodular pattern and multiple adenopathies. CT: computed tomography.

veolar lavage (BAL) and bronchial lavage showed a negative direct test for acid-fast bacilli (AFB) on both. At day 4, we were notified that an IGRA screening test performed a few weeks earlier was positive.

\section{Treatment}

He was started on isoniazid, rifampin, pyrazinamide and ethambutol. Seven days later, the BAL cultural exam revealed AFB and DNA testing was positive for MB. As of such, we related the abdominal CT findings to peritoneal TB involvement.

At day 17, he started complaining of memory loss and mental slowing. Neurological examination was unremarkable, but brain contrast $\mathrm{CT}$ revealed multiple small rounded scattered contrast-enhancing lesions. Magnetic resonance imaging (MRI) confirmed numerous intra-axial cortico-subcortical lesions with ring enhancement after contrast, with about $5 \mathrm{~mm}$ of major axis, dispersed throughout both cerebral hemispheres

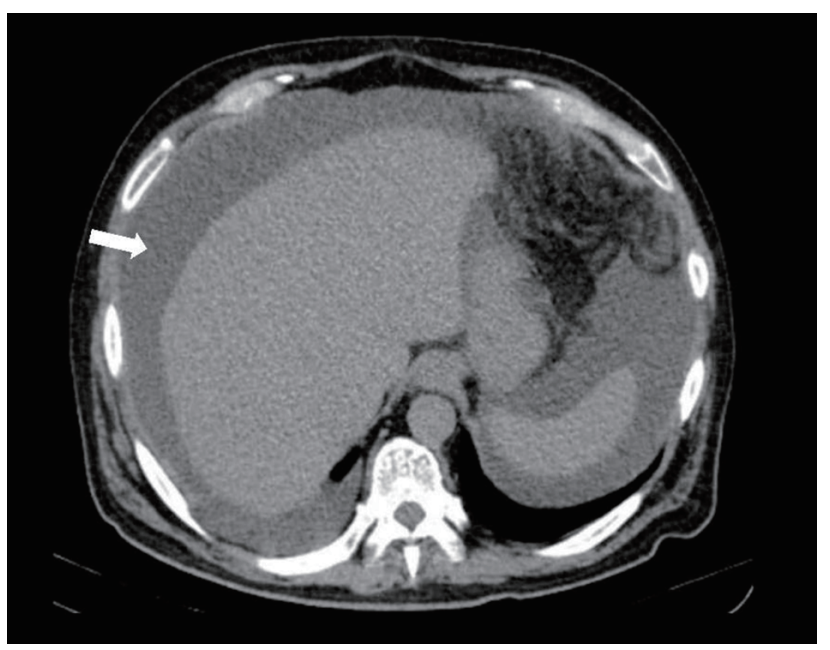

Figure 2. Abdominal-pelvic CT revealing high-volume ascites. CT: computed tomography.

that exhibit slight restriction to peripheral diffusion, with surrounding vasogenic edema, suggestive of tuberculomas (Fig. 3 ). Lumbar puncture showed 23 leukocytes $/ \mu \mathrm{L}$, with predominance of mononuclear cells, $84 \mathrm{mg} / \mathrm{dL}$ proteinorachie, with normal glucose levels. Cerebrospinal fluid (CSF) ADA, MB DNA and AFB were negative.

Assuming central nervous system disseminated TB, corticosteroid therapy ( $1 \mathrm{mg} / \mathrm{kg}$ of prednisolone) was initiated for this purpose. There was no evidence of cardiac involvement on transthoracic echocardiography.

\section{Follow-up and outcomes}

The patient began to improve, maintaining apyrexia after day 18 . The remaining study showed a negative rifampin resistance test. He completed a 12-month treatment with the anti-TB drugs with weaning corticosteroid therapy. The clinical outcome was good and the patient did not restart adalimumab therapy.

\section{Discussion}

TB can present with a variable clinical picture making the diagnosis difficult [1]. Disseminated TB is defined as having two or more non-contiguous sites resulting from lymphohematogenous dissemination of MB [1]. Extrapulmonary involvement occurs in one-fifth of all TB cases and it may occur in the absence of histological and radiological evidence of pulmonary infection [1].

Anti-TNF- $\alpha$ drugs emerged as an effective treatment in immune-mediated inflammatory diseases (IMID) such as rheumatoid arthritis, ankylosing spondylitis and psoriatic arthritis [2]. The immunosuppressive effect of the drugs contributes to a TB increased incidence around the world. The risk of TB associated with the different biological therapies varies significantly, with the highest relative risk, 29.3 and 18.6, associated with adalimumab and infliximab, respectively [3]. 


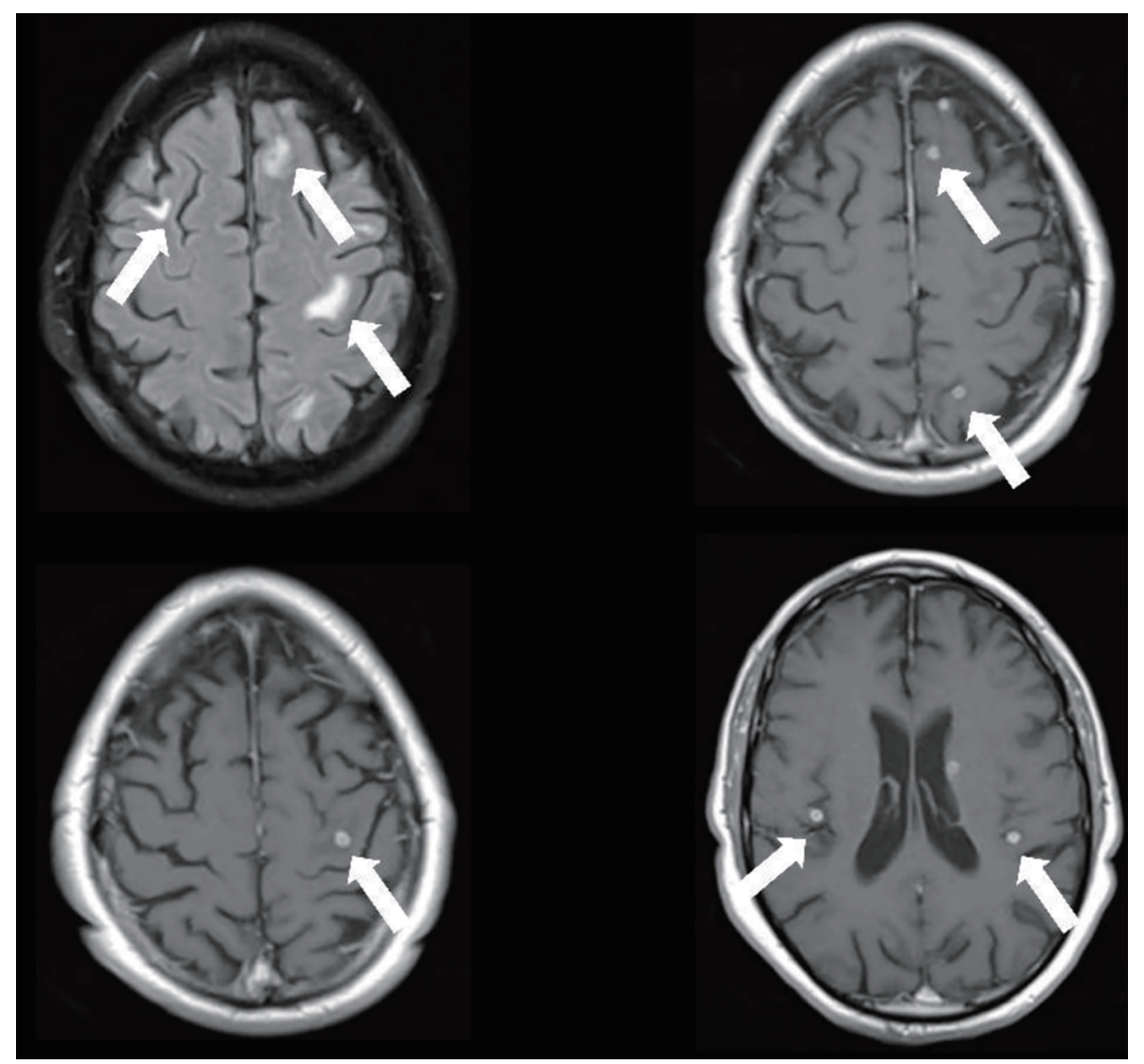

Figure 3. Cerebral MRI depicting numerous intra-axial cortico-subcortical lesions with ring enhancement after contrast, dispersed throughout both cerebral hemispheres with surrounding vasogenic edema, suggestive of tuberculomas. MRI: magnetic resonance imaging.

However, TNF- $\alpha$ is a key cytokine in protecting host defence against MB [2]. Most cases occur at the beginning of treatment and the reactivation usually has a rapid progression being sometimes fatal. As of such, many experts state that individuals with risk factors for TB should be under isoniazid prophylaxis in the 3 months after initiating therapy. When TB infection does develop in patients treated with anti-TNF- $\alpha$ drugs, anti-TB agents must be started promptly. Immunosuppression is usually stopped in the beginning of TB treatment but should be restarted as soon as possible if still indicated.

In this report, we came across a patient with longstanding adalimumab immunosuppression, with recent negative latent TB screening who presented with disseminated TB. Performance of latent TB screening tests is affected by IMID and immunosuppressive therapy in patients due to commence TNF- $\alpha$ inhibitor treatment [3]. Latent TB screening programs prior to the treatment significantly reduce the incidence of $\mathrm{TB}$, but the optimal screening algorithm, in particular the question of whether a combination of IGRA and TST or a single test only should be used, is a matter of ongoing debate [3]. Until recently, TST has been the only diagnostic method for latent TB, however its specificity is low [4]. IGRA is more specific, its technique is simpler and faster than TST and it is not affected by prior BCG vaccination nor most non-tuberculous mycobacteria infections [4]. IGRA in combination with TST is used to increase sensitivity [3].

Several authors defend the need for annual monitoring of latent TB with IGRA, but this patient had a negative test the year before he started anti-TNF- $\alpha$ therapy and another negative test 5 years later under the same therapy. As such, patient TB symptom awareness is of the utmost importance for early diagnosis, especially in geographic high-risk locations.

In conclusion, TB still remains a serious public health problem worldwide. As it may mimic several conditions, a high index of suspicion may be necessary for diagnosis. Disseminated TB is unlikely in immunocompetent patients. The risk increases under certain conditions like alcohol abuse, human immunodeficiency virus, diabetes mellitus and hematologic diseases.

\section{Acknowledgments}

None to declare.

\section{Financial Disclosure}

None to declare. 


\section{Conflict of Interest}

None to declare.

\section{Informed Consent}

Both written and verbal informed consents were obtained from the patient for publication of this case report.

\section{Author Contributions}

Marinha Silva: literature review, final manuscript writing. Joana Braga: literature review, initial manuscript draft. Cristiana Fernandes: literature review, initial manuscript draft. Jose Miguel Ferreira: literature review, collected patient data. Cristina Marques: literature review, collected patient data. Carina Costa Silva: literature review, initial manuscript draft. Carla Lemos Costa: final manuscript writing.

\section{Data Availability}

The authors declare that data supporting the findings of this study are available within the article.

\section{References}

1. Ribeiro S, Trabulo D, Cardoso C, Oliveira A, Cremers I. Disseminated tuberculosis in an immunocompetent patient: the answer is in the liver. GE Port J Gastroenterol. 2016;23(4):208-213.

2. Lalvani A, Millington KA. Screening for tuberculosis infection prior to initiation of anti-TNF therapy. Autoimmun Rev. 2008;8(2):147-152.

3. Dobler CC. Biologic Agents and Tuberculosis. Microbiol Spectr. 2016;4(6):4.6.49.

4. Harada N. [Characteristics of a diagnostic method for tuberculosis infection based on whole blood interferongamma assay]. Kekkaku. 2006;81(11):681-686. 